\title{
Characterization of UDP-Glucuronosyltransferases and the Potential Contribution to Nicotine Tolerance in Myzus persicae
}

\author{
Yiou Pan ${ }^{1,2}$, Pengjun $\mathrm{Xu}^{3}$, Xiaochun Zeng ${ }^{1}$, Xuemei Liu ${ }^{1}$ and Qingli Shang ${ }^{1,2, *(\mathbb{B})}$ \\ College of Plant Science, Jilin University, Changchun 130062, China \\ 2 School of Agricultural Science, Zhengzhou University, Zhengzhou 450001, China \\ 3 Institute of Tobacco Research, Chinese Academy of Agricultural Sciences, Qingdao 266101, China \\ * Correspondence: shangqingli@163.com
}

Received: 28 June 2019; Accepted: 22 July 2019; Published: 25 July 2019

check for updates

\begin{abstract}
Uridine diphosphate (UDP)-glycosyltransferases (UGTs) are major phase II detoxification enzymes involved in glycosylation of lipophilic endobiotics and xenobiotics, including phytoalexins. Nicotine, one of the most abundant secondary plant metabolites in tobacco, is highly toxic to herbivorous insects. Plant-herbivore competition is the major impetus for the evolution of large superfamilies of UGTs and other detoxification enzymes. However, UGT functions in green peach aphid (Myzus persicae) adaptation are unknown. In this study, we show that UGT inhibitors (sulfinpyrazone and 5-nitrouracil) significantly increased nicotine toxicity in M. persicae nicotianae, suggesting that UGTs may be involved in nicotine tolerance. In total, 101 UGT transcripts identified in the M. persicae genome/transcriptome were renamed according to the UGT Nomenclature Committee guidelines and grouped into 11 families, UGT329, UGT330, UGT339, UGT341-UGT345, and UGT348-UGT350, with UGT344 containing the most (57). Ten UGTs (UGT330A3, UGT339A2, UGT341A6, UGT342B3, UGT343C3, UGT344D5, UGT344D8, UGT348A3, UGT349A3, and UGT350A3) were highly expressed in $M$. persicae nicotianae compared to $M$. persicae sensu stricto. Knockdown of four UGTs (UGT330A3, UGT344D5, UGT348A3, and UGT349A3) significantly increased M. persicae nicotianae sensitivity to nicotine, suggesting that UGT expression in this subspecies may be associated with nicotine tolerance and thus host adaptation. This study reveals possible UGTs relevant to nicotine adaptation in tobacco-consuming $M$. persicae nicotianae, and the findings will facilitate further validation of the roles of these UGTS in nicotine tolerance.
\end{abstract}

Keywords: UDP-glucuronosyltransferase; host adaptation; Myzus persicae; nicotine tolerance

\section{Introduction}

The peach potato or green peach aphid Myzus persicae Sulzer (Hemiptera: Aphididae) is a globally important pest that affects a broad range of agricultural and horticultural crops, causing significant damage both through direct feeding and transmission of many plant viruses [1]. The ability of $M$. persicae to adapt to new host plants has led to the formation of host races. The best documented case of this phenomenon is the adaptation of M. persicae to the host plant tobacco (Nicotiana tabacum L.); this led to the host race designated M. persicae nicotianae [2]. M. persicae nicotianae is morphologically and genetically distinct from $M$. persicae sensu stricto (s.s.). However, there are clear examples of gene flow between the two taxa [3]. In another case, M. persicae clones collected from Western Australia have adapted to feed successfully on Lupinus angustifolius (narrow-leafed lupine), and this adaptation might be due to enhanced tolerance to lupanine in the diet compared to that of non-adapted clones [4].

Plants produce many toxic secondary metabolites, some of which are thought to act in direct defense against herbivores by reducing their performance, survival, and reproduction. 
Furthermore, phytophagous insects have evolved various strategies to cope with allelochemicals, including detoxification enzymes, such as cytochrome P450 monooxygenases (P450), carboxylesterases (CarE), glutathione S-transferases (GSTs), and uridine diphosphate (UDP)-glycosyltransferases (UGTs) [5-7]. Various reports have shown that P450s of the CYP3 clade are involved in oxidative detoxification of furanocoumarins, alkaloids, and numerous other plant secondary metabolites and synthetic insecticides [8-12]. Nonetheless, the roles of UGTs in xenobiotic tolerance have not been sufficiently studied.

UGTs catalyze conjugation of a diverse range of small lipophilic xenobiotics and endobiotics with sugars to produce glycosides, which are water-soluble and can be efficiently excreted [13]. Therefore, glycosylation of toxins by UGTs is a particularly important detoxification mechanism [14,15]. However, only a few reports to date have indicated the involvement of insect UGTs in the detoxification of plant secondary xenobiotics, such as in Manduca sexta [16], Helicoverpa assulta [17,18], Spodoptera littoralis [19], and Helicoverpa armigera [20]. It has recently been demonstrated that increased expression and amplification of the $\mathrm{CYP} 6 \mathrm{CY} 3$ gene in $M$. persicae nicotianae plays a major role in detoxifying nicotine and that 2-hydroxynicotine and 5-hydroxynicotine are produced by hydroxylation reactions [21,22]. UGTs usually catalyze the transfer of glycosyl groups of sugar donors (e.g., UDP-glucose) to hydroxyl/amino groups of lipophilic compounds to produce glycosides $[19,20]$, yet it remains unknown whether abundant UGTs are involved in nicotine tolerance via secondary detoxification metabolism in $M$. persicae nicotianae.

To advance our understanding of the potential roles of UGTS in nicotine tolerance in M. persicae nicotianae, the following experiments were performed: (1) UGT transcripts were identified in the M. persicae genome and transcriptome; (2) using quantitative real-time polymerase chain reaction (qRT-PCR), the expression profiles of UGTs that were identified in the transcriptome were analyzed in three M. persicae clones; and (3) the involvement of UGTs in nicotine tolerance in M. persicae nicotianae was functionally confirmed by RNA interference (RNAi) assays. In the present study, we potentially identified putative UGT transcripts in this subspecies and assigned standard names according to the guidelines of the UGT Nomenclature Committee. Our data provide preliminary insight into changes in UGTs' expression in three M. persicae clones based on transcriptome data and their involvement in nicotine tolerance. These results may facilitate further study of the functions of UGTs in M. persicae with regards to host plant adaptation.

\section{Results}

\subsection{Synergism Bioassays}

Two UGT inhibitors, sulfinpyrazone (Sul) and 5-nitrouracil (5-Nul), were used to analyze the possible roles of UGTs in nicotine tolerance. The results of the synergistic effects of 5-Nul and Sul on nicotine toxicity in M. persicae nicotianae are presented in Figure 1. 5-Nul and Sul possibly increased nicotine toxicity in apterous adult M. persicae nicotianae; mortality increased from $49.07 \%$ under nicotine treatment alone $(100 \mathrm{mg} / \mathrm{L})$ to $65.86 \% / 66.13 \%$ under nicotine treatment $(100 \mathrm{mg} / \mathrm{L})$ with $5-\mathrm{Nul} / \mathrm{Sul}$ $(12.5 \mathrm{mg} / \mathrm{L})$ treatment (Figure 1). These results indicate that UGTs may be involved in nicotine tolerance in M. persicae nicotianae. 


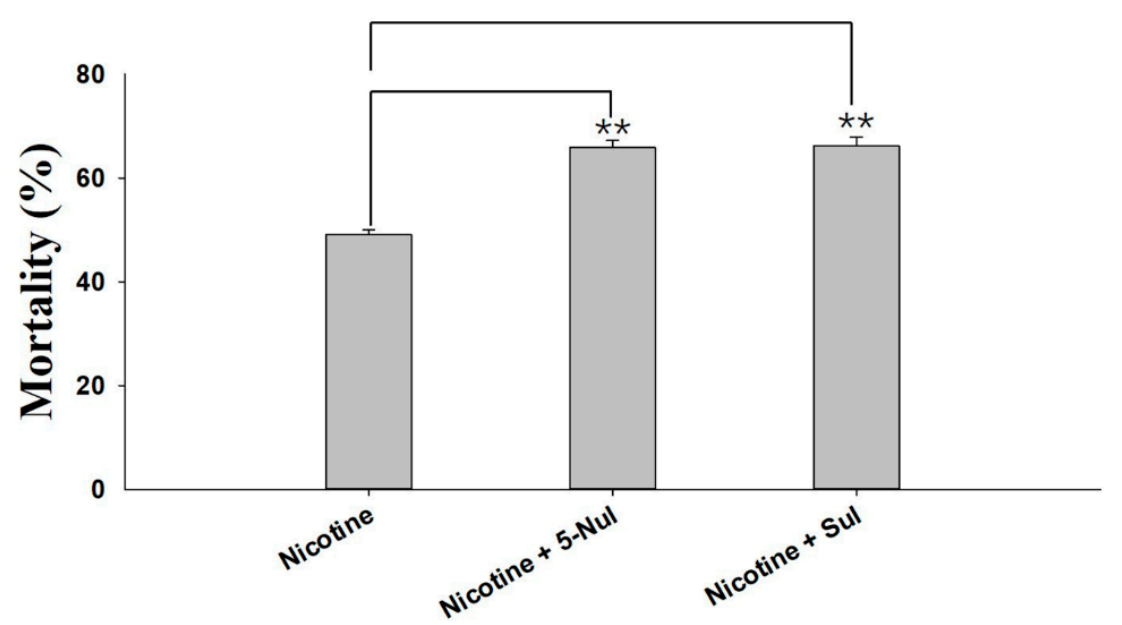

Figure 1. Synergistic effects of sulfinpyrazone (Sul) and 5-nitrouracil (5-Nul) on the toxicity of nicotine in M. persicae nicotianae. The final concentrations of nicotine, Sul, and 5-Nul in the artificial diet were $100 \mathrm{mg} / \mathrm{L}, 12.5 \mathrm{mg} / \mathrm{L}$, and $12.5 \mathrm{mg} / \mathrm{L}$, respectively. Mortality was recorded after $48 \mathrm{~h}$ of treatment (three replicates, 80 apterous adult aphids per replicate). Error bars indicate $95 \%$ confidence intervals $(n=3)$. ** Significant difference according to Student's $t$-test $(p<0.01)$.

\subsection{Identification and Phylogenetic Analysis of M. persicae UGTs}

Based on the transcriptome data for M. persicae (the clean reads obtained in this study were submitted to the NCBI/Sequence Read Archive (SRA) database under the SRA experiment Accession Number SRX1499035), 39 UGT transcripts with full-length or nearly full-length open reading frames (ORF) were identified, and these UGTs were named according to the UGT Nomenclature Committee (http://prime.vetmed.wsu.edu/resources/udp-glucuronsyltransferase-homepage) guidelines to include the following components: the symbol UGT, a family number, a subfamily letter, and an individual gene number. UGT families are defined as having $40 \%$ amino acid sequence identity, and subfamilies are defined as having $60 \%$ or greater amino acid identity [23]. The GenBank accession numbers are listed in Supplementary Data 1. Ninety UGT transcripts in the M. persicae genome were downloaded from the National Center for Biotechnology Information (NCBI, https://www.ncbi.nlm.nih.gov/nucleotide/) and named according to the UGT Nomenclature Committee guidelines; these UGT sequences were identified as representing 71 UGT transcripts. In total, 101 UGT transcripts were identified in M. persicae after removing redundant sequences (Supplementary Data 1). Phylogenetic analysis was used to evaluate the evolutionary relationships of UGTs. UGT sequences from H. armigera and Bombyx mori in the UGT Nomenclature Committee database (Supplementary Data 2) and UGT gene sequences from M. persicae were used to construct a phylogenetic tree (Figure 2). ClustalW was employed to align the amino acid sequences in MEGA7 software (http://www.megasoftware.net/), and the neighbor-joining method with 1000 bootstrap replicates was applied for construction of the phylogenetic tree. Bootstrap values above $50 \%$ are indicated on the branches.

The 101 M. persicae UGT transcripts were distributed into 11 families. Of the total, 57 UGTs were grouped into the UGT344 family; 16 into the UGT343 family; 7 each into the UGT329 and UGT350 families; 4 into the UGT341 family; 3 into the UGT342 family; 2 each into the UGT330 and UGT348 families; and only one each into the UGT339, UGT345, and UGT349 families (Figure 2, Supplementary Data 1). The gene evolution relationships of H. armigera/B. mori and M. persicae's UGTs are far from each other. Because the roles of UGTs in xenobiotic compound tolerance are still poorly understood, we performed further analyses. 


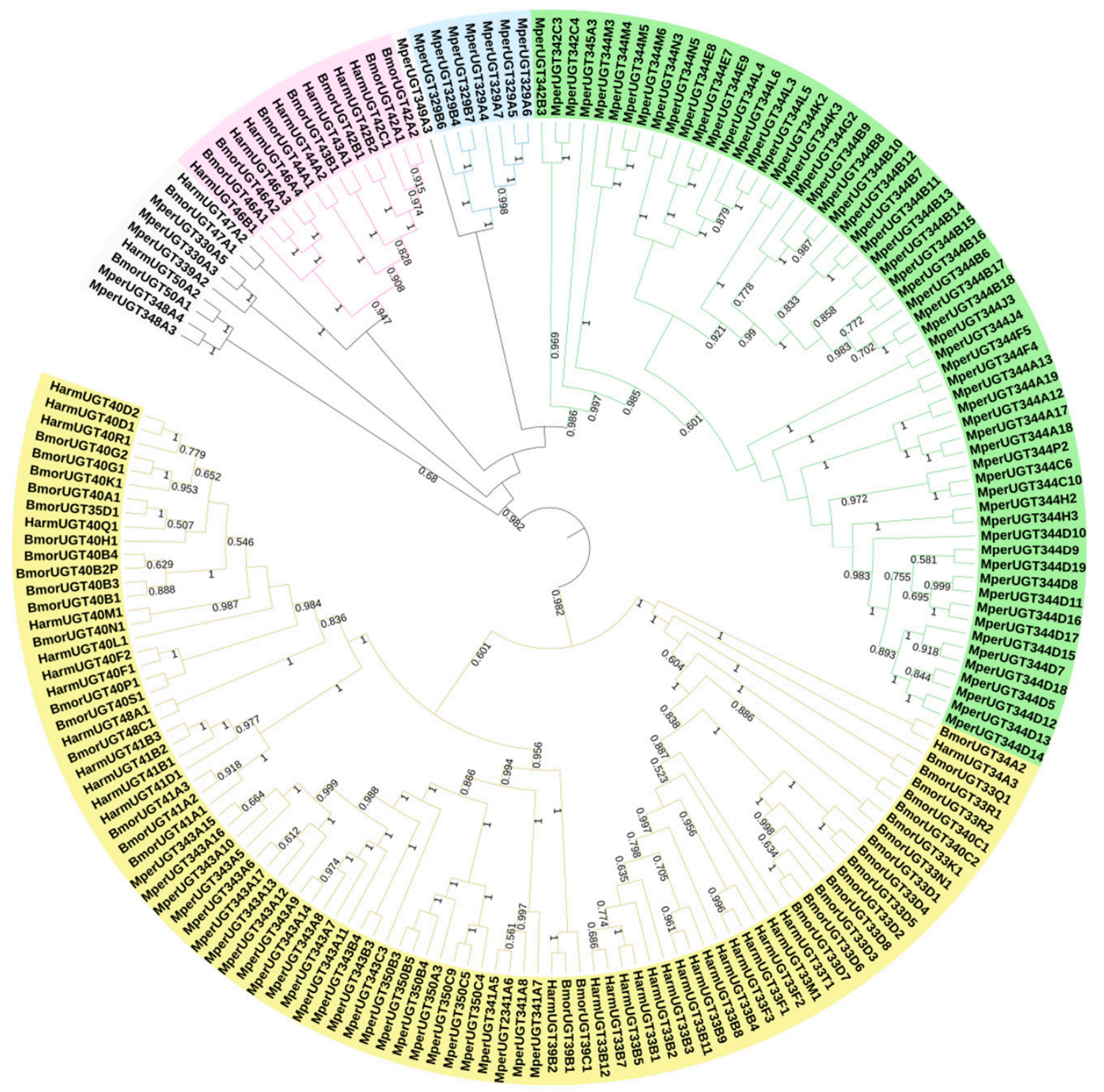

Figure 2. Phylogenetic analysis of M. persicae UGT genes and orthologs in H. armigera and B. mori. A phylogram was generated using the maximum likelihood method in MEGA7, and bootstrap values were computed based on 1000 replicates with a cutoff of $<50 \%$. The sequences used for constructing the tree are listed in Supplementary Data 1 and Supplementary Data 2.

\subsection{Conserved Domains of the M. persicae UGT Proteins}

Multiple alignments of eight representative M. persicae UGT amino acid sequences revealed two major domains: a highly-variable N-terminal substrate-binding domain and a conserved C-terminal sugar donor-binding domain (Figure 3) [18]. All the UGTs contained a variable-length amino acid signal peptide found at the N-terminus, which was presumably cleaved after integration into the endoplasmic reticulum (ER) compartment. The two predicted sugar donor-binding regions (DBR1 and DBR2) and important residues interacting with the sugar donor and catalytic residues were conserved. The UGT motif signature sequence, (FVA)-(LIVMF)-(TS)-(HQ)-(SGAC)-G-X (2)-(STG)-X(2)-(DE)-X(6)-P-(LIVMFA)-(LIVMFA)-X(2)-P-(LMVFIQ)-X(2)-(DE)-Q, is present in the middle of the $\mathrm{C}$-terminal domain, which shows high conservation. The alignment data suggest that these M. persicae UGT proteins may have glycoside conjugation activity. 


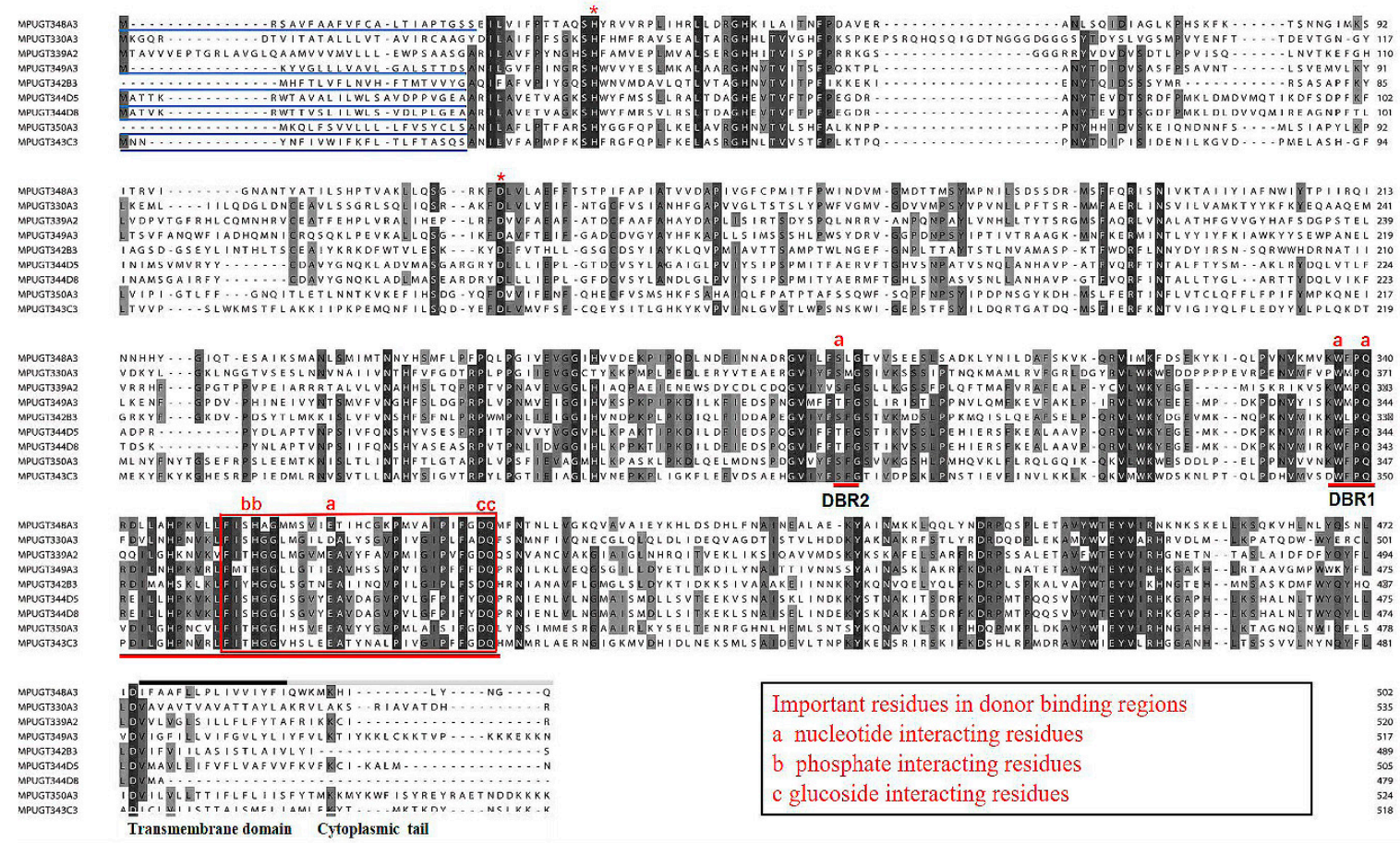

Figure 3. Alignment of the amino acid sequences of nine UGT genes from M. persicae. The predicted signal peptides in the N-terminus are underlined. The UGT signature motif is boxed. The transmembrane domains in the C-terminal half and the cytoplasmic tails are indicated with black and gray lines above the alignment. The important catalytic residues, $\mathrm{H}$ and $\mathrm{D}$, are indicated with asterisks $\left({ }^{*}\right)$ above the alignment. The red lines under the alignment indicate the two donor-binding regions (DBRs), and several residues interacting with the sugar donor are indicated by letters $(a, b$, and c) above the alignment.

\subsection{Differences in UGT Gene Expressions among Three M. persicae Subspecies}

qRT-PCRs were employed to determine the mRNA expression levels of 37 UGTs identified in the transcriptomes of three M. persicae subspecies. Expression of nine UGTs (UGT330A3, UGT339A2, UGT342B3, UGT343C3, UGT344D5, UGT344D8, UGT348A3, UGT349A3, and UGT350A3) was significantly higher in M. persicae nicotianae (green) than in M. persicae sensu stricto (green) and M. persicae sensu stricto (red) (Figure 4). Additionally, expression of UGT341A6 was 0.62- and 14.55-fold higher in M. persicae nicotianae (green) than in M. persicae sensu stricto (green) and M. persicae sensu stricto (red), respectively. With regard to the other UGTs, levels were less significantly different or were not significantly different among the aphids (Figure 4).

\subsection{Knockdown of UGT Transcripts Increases Mortality in M. persicae nicotianae}

RNAi experiments using orally-delivered dsRNA were performed to elucidate the relationship between downregulation of highly-overexpressed UGTs and mortality, which could be a result of nicotine intolerance. Ten UGT genes highly expressed in M. persicae nicotianae (UGT330A3, UGT339A2, UGT341A6, UGT342B3, UGT343C3, UGT344D5, UGT344D8, UGT348A3, UGT349A3, and UGT350A3) according to the qRT-PCRs (Figure 4) were chosen for the RNAi experiments. After dsRNA treatment (100 ng/ $\mu \mathrm{L}$ dsRNA-UGT), the expression levels of UGT330A3, UGT341A6, UGT342B3, UGT343C3, UGT344D5, UGT344D8, UGT348A3, and UGT349A3 were reduced by 0.50-, 0.54-, 0.46-, 0.58-, 0.34-, $0.40-, 0.64-$, and 0.47 -fold after $48 \mathrm{~h}$ compared to the levels in the control group (Figure 5). Conversely, expression of UGT339A2 and UGT350A3 was not affected by oral delivery of dsRNA (Figure 5). Mortality under $100 \mathrm{mg} / \mathrm{L}$ nicotine treatment significantly increased after RNAi, from $50.10 \%$ in the control group to $61.47 \%$ and $71.65 \%$ in the $d s R N A-U G T 330 A 3$ - and $d s R N A-U G T 348 A 3$-fed groups, respectively (Figure 6). Moreover, suppression of UGT344D5 and UGT349A2 expression dramatically increased 
mortality from $45.03 \%$ in the control group to $54.31 \%$ and $59.98 \%$ in the $d s R N A-U G T 344 D 5-$ and dsRNA-UGT349A2-treated groups, respectively, and mortality under $100 \mathrm{mg} / \mathrm{L}$ nicotine treatment was highest (64.91\%) among aphids fed a dsRNA mixture (with equal amounts of the eight dsRNA-UGT-Mix) (Figure 6).
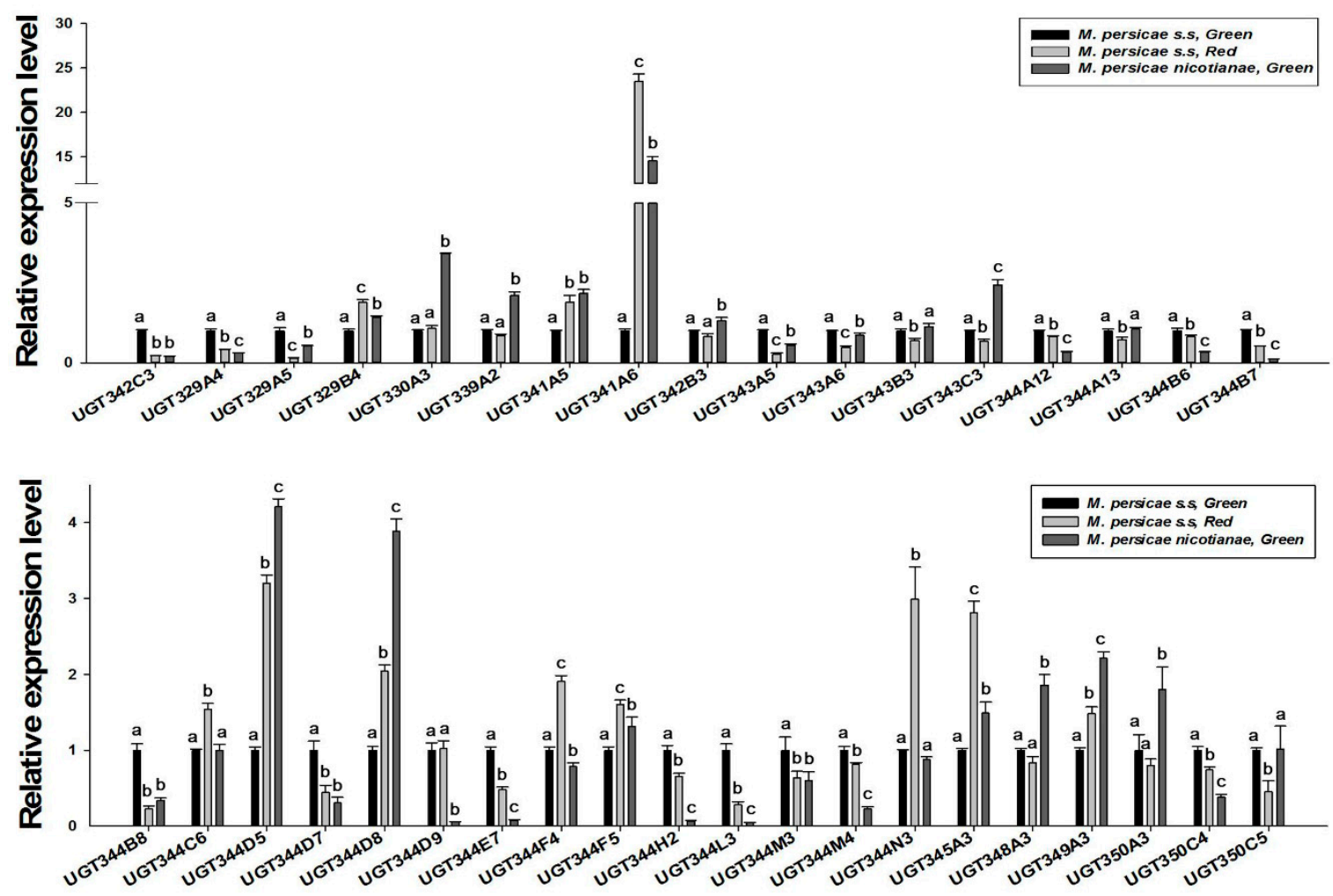

Figure 4. Relative expression levels of UGTs in three populations of M. persicae determined by real-time PCR. Actin and para (a voltage-gated sodium channel) were used as internal reference genes. Error bars indicate $95 \%$ confidence intervals $(n=3)$. Different letters on the bars indicate significant differences based on ANOVA followed by Tukey's HSD multiple comparison test $(p<0.05)$.

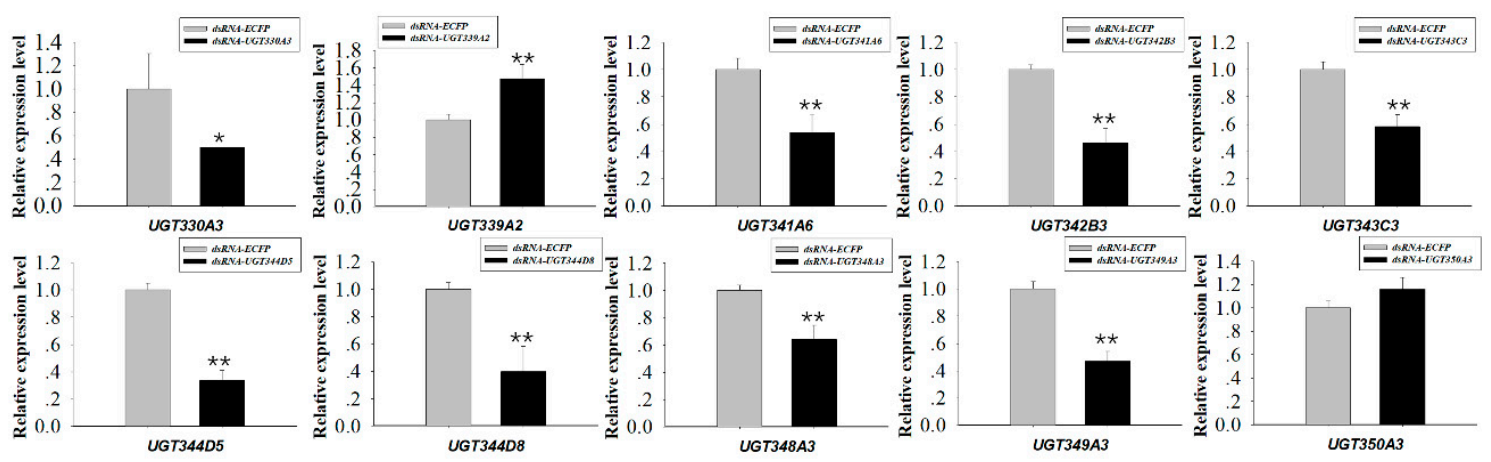

Figure 5. Relative expression of 10 UGT transcripts in M. persicae nicotianae. dsRNA-mediated $48 \mathrm{~h}$ after oral delivery of dsRNA. Suppression of UGT (UGT330A3, UGT339A2, UGT341A6, UGT342B3, UGT343C3, UGT344D5, UGT344D8, UGT348A3, UGT349A3, and UGT350A3) expression in M. persicae nicotianae fed an artificial diet with corresponding dsRNA (100 ng/ $\mu \mathrm{L})$ for $48 \mathrm{~h}$. Error bars indicate $95 \%$ confidence intervals $(n=3)$. ${ }^{*}$ Significant $(p<0.05)$ difference according to Student's $t$-test. ** Significant $(p<0.01)$ difference according to Student's $t$-test. 

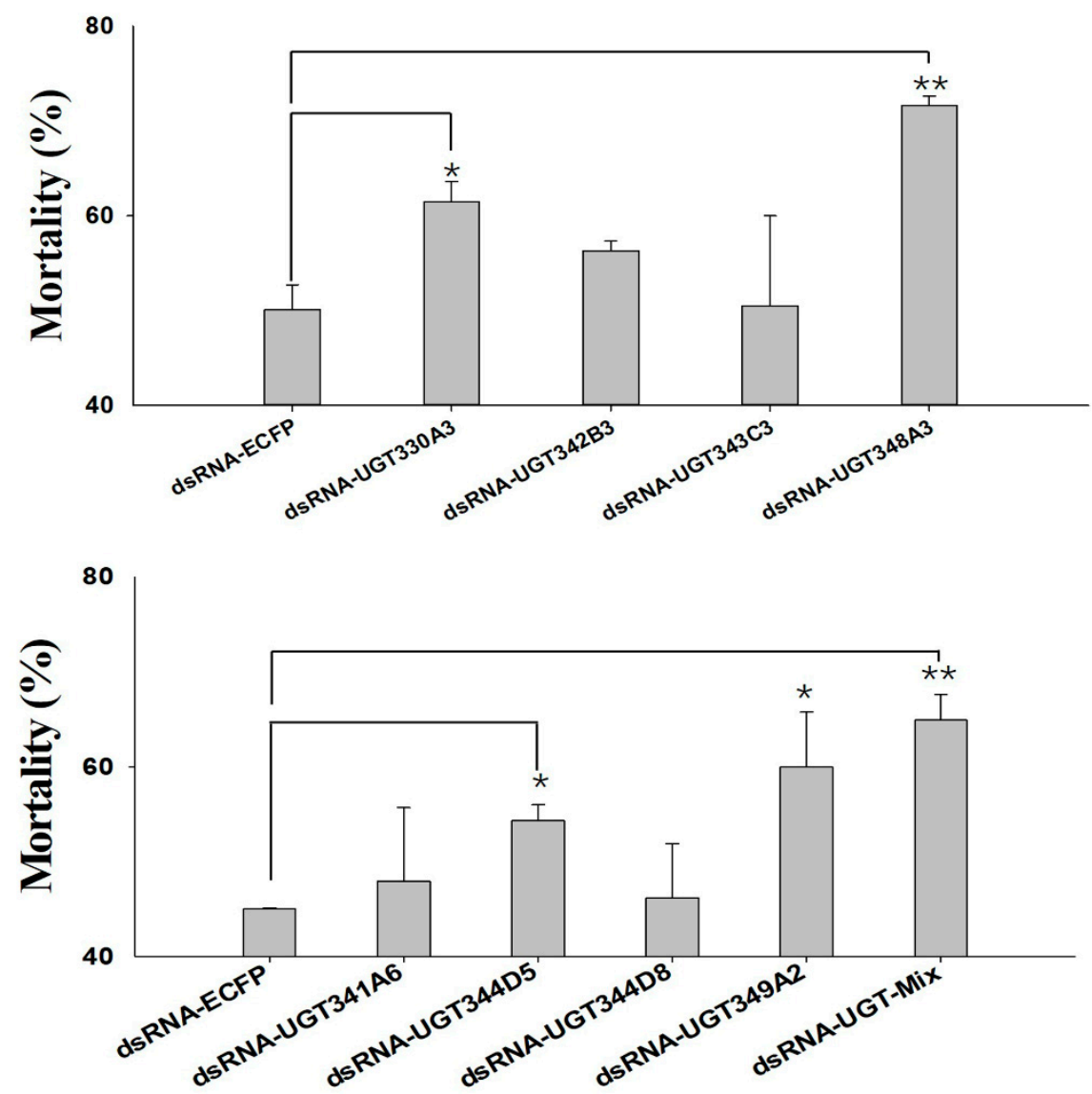

Figure 6. Effects of knockdown of UGT transcripts on mortality of M. persicae nicotianae. Mean mortality \pm SE $(n=3)$ of M. persicae nicotianae fed nicotine $(100 \mathrm{mg} / \mathrm{L})$ and a dsRNA-UGT $(100 \mathrm{ng} / \mu \mathrm{L})$ for $48 \mathrm{~h}$. Each treatment included three replicates, and 80 adult $M$. persicae nicotianae were used in each replicate. * Significant $(p<0.05)$ difference according to Student's $t$-test. ${ }^{* *}$ Significant $(p<0.01)$ difference according to Student's $t$-test.

\section{Discussion}

Host plants accumulate phytotoxins, such as nicotine in Nicotiana tabacum, to resist insect herbivores, and aphid survival in the presence of toxic secondary metabolite nicotine depends on nicotine metabolism. Indeed, metabolism is essential for insects to counter the effects of toxins distributed in host plants. Research has indicated that higher expression of the phase I enzyme P450-CYP6CY3 in M. persicae nicotianae versus M. persicae sensu stricto, which is partly due to gene amplification, accounts for the nicotine and neonicotinoid resistance of the subspecies [21]. In the cotton bollworm, larval tolerance to gossypol depends on CYP6AE14-mediated detoxification [11,24]. Recent studies have also indicated that the phase II enzymes UGT41B3 and UGT40D1 are capable of glycosylating gossypol to form mainly diglycosylated gossypol isomer 5 , accounting for gossypol tolerance in H. armigera [20]. In addition, stereoselective reglucosylation of the benzoxazinoid DIMBOA by UGTs represents a detoxification strategy for the adaptation of Spodoptera species to benzoxazinoid-containing plants [19]. Although the roles of P450 in nicotine tolerance in M. persicae nicotianae have been described [21], the potential roles of UGTs in nicotine tolerance remain unknown. To clarify the roles of these enzymes in host plant adaptation in M. persicae nicotianae, two UGT inhibitors, 5-Nul and Sul, were used in a synergism assay. 5-Nul/Sul significantly promoted nicotine toxicity in M. persicae nicotianae (Figure 1), illustrating that UGTs may be involved in nicotine tolerance. Therefore, glycosylation by UGTs may play an important role in the detoxification of xenobiotics or the primary metabolites of xenobiotics in M. persicae nicotianae. However, information regarding UGTs in M. persicae is very limited to date. 
In this study, 101 UGT transcripts were identified from the M. persicae genome and transcriptome data. These UGTs can be distributed into 11 families, UGT330A3, UGT339A2, UGT341A6, UGT342B3, UGT343C3, UGT344D5, UGT344D8, UGT348A3, UGT349A3, and UGT350A3, with the UGT344 family containing the most transcripts (57 UGTs) (Figure 2). The UGT protein structure is divided into two main parts: an aglycone substrate-binding domain in the N-terminus and a UDP sugar donor-binding domain in the C-terminus [23,25]. Alignment of UGT amino acid sequences revealed conserved domains, including sugar donor-binding regions (DBR1 and DBR2), important residues interacting with the sugar donor and catalytic residues, and the UGT signature motif sequence ((FVA)-(LIVMF)-(TS)-(HQ)-(SGAC)-G-X(2)-(STG)-X(2)-(DE)-X(6)-P-(LIVMFA)-(LIVMFA)-X(2)-P(LMVFIQ)-X(2)-(DE)-Q) (Figure 3) [13]. These results suggest that these UGTs are likely active proteins that function in M. persicae as they do in other species.

Insect UGTs are not only capable of detoxifying plant secondary compounds [19,20], some studies have also indicated that UGTs are involved in insecticide resistance [26-28]. For example, UGT overexpression is associated with imidacloprid and abamectin resistance in Leptinotarsa decemlineata and Tetranychus cinnabarinus, respectively $[29,30]$. However, there has thus far been no large-scale screening of potential xenobiotic tolerance-associated UGTs in M. persicae. In the present study, qRT-PCR analysis of the transcriptional profiles of UGTs based on transcriptome data revealed that the expression levels of ten UGT genes was consistent with the levels of nicotine tolerance in the M. persicae sensu stricto (green/red morph) and M. persicae nicotianae populations (Figure 4), suggesting the potential roles of these UGTS in host plant adaptation in M. persicae nicotianae. In vivo suppression of UGT gene expression by RNAi was employed to examine the functions of UGTs in xenobiotic compound resistance. In previous studies, knockdown of highly-expressed UGT increased the susceptibility of resistant Leptinotarsa decemlineata to imidacloprid [29]. In Plutella xylostella, RNAi of transcript UGT2B17 (renamed UGT33AA4) showed this gene to be associated with chlorantraniliprole resistance [26]. To assess the influence of strong UGT gene expression on nicotine tolerance in M. persicae nicotianae, we performed RNAi via the dsRNA oral feeding method [31]. RNAi of the four highly-expressed UGT genes (UGT330A3, UGT344D5, UGT348A3, and UGT349A3) alone and a mixture of eight dsRNA of UGTs significantly increased susceptibility to nicotine in M. persicae nicotianae (Figures 5 and 6). This result further confirmed that the enzymes encoded by these strongly-expressed UGTs (UGT330A3, UGT344D5, UGT348A3, and UGT349A3) may contribute to detoxification of nicotine or its primary metabolites via glycosylation and may further contribute to host plant adaptation in $M$. persicae nicotianae. These results further our understanding of host adaptation mechanisms in M. persicae.

\section{Materials and Methods}

\subsection{Insects}

Three M. persicae clone populations were established from a population that was originally collected in 2008 from a field in Jilin Province, China. An M. persicae sensu stricto population (green/red morph) was sampled and reared on Chinese cabbage (Brassica rapa chinensis). An M. persicae nicotianae population (green morph) was collected and reared on tobacco (N. tabacum L.). All populations were reared in the laboratory at $20^{\circ} \mathrm{C}-23^{\circ} \mathrm{C}$ with a photoperiod of $16 \mathrm{~h}: 8 \mathrm{~h}$ (light:dark).

\subsection{Synergism Assays}

The synergistic effects of the two UGT inhibitors Sul and 5-Nul on the toxicity of nicotine in M. persicae nicotianae were tested using the artificial diet method, as described by Peng et al. (2016) [31]. The artificial diet (15\% sucrose) was sealed between two layers of Parafilm in a $4-\mathrm{cm}$ feeding arena. Aphids were placed in the arena, which was covered with fine mesh to prevent their escape. The maximum sublethal doses of Sul/5-Nul in M. persicae nicotianae were determined using the oral feeding method. At least five different concentrations of Sul/5-Nul and a control were used. The maximum dose that led to zero mortality in M. persicae nicotianae was adopted as the maximum sublethal 
concentration in our study. Eighty apterous adult M. persicae nicotianae were fed an artificial diet that contained nicotine (final concentration: $100 \mathrm{mg} / \mathrm{L}$ ) with or without added Sul/5-Nul (final concentration: $12.5 \mathrm{mg} / \mathrm{L}$ ); the artificial diet without inhibitor was used as a control. Each treatment included three replicates (80 apterous adults in each replicate). Mortality was recorded after $48 \mathrm{~h}$. Synergistic effects were calculated by comparing the corrected mortality without Sul/5-Nul to the corrected mortality with Sul/5-Nul.

\subsection{Nomenclature and Phylogenetic Analysis}

UGT sequences from the genome of M. persicae in NCBI and the transcriptome of M. persicae (the SRA experiment Accession Number SRX1499035) were obtained [31]. These UGT sequences were named according to the UGT Nomenclature Committee (http://prime.vetmed.wsu.edu/resources/udpglucuronsyltransferase-homepage) guidelines to include the following components: the symbol UGT, a family number, a subfamily letter, and an individual gene number. UGT families are defined as having $40 \%$ amino acid sequence identity, and subfamilies are defined as having $60 \%$ or greater amino acid identity [23]. Names were assigned to the M. persicae UGT sequences on this basis (Supplementary Data 1). Predicted UGT protein sequences from H. armigera and B. mori (Supplementary Data 2) were extracted from UGT Nomenclature Committee resources and aligned against $M$. persicae UGTs using ClustalW in MEGA7 software (http://www.megasoftware.net/). These alignments were used to build a consensus phylogenetic tree with the neighbor-joining method. Pairwise and multiple alignments were performed with a gap opening penalty of 10 and a gap extension penalty of 0.2 . A total of 1000 bootstrap replicates were used. Branches with bootstrap values above $50 \%$ are indicated.

\subsection{Protein Structure Prediction}

Multiple alignments of nine highly-expressed representative protein sequences among the M. persicae nicotianae UGTs were performed using ClustalW, and structural domains such as the signature UGT motif were detected by comparison with other sequences with characterized primary structures. Signal peptides were predicted by SignalP 4.1 on CBS Prediction Servers (http://www.cbs. dtu.dk/services/SignalP/). The C-terminal transmembrane domain was identified with TMHMM2.0 (http://www.cbs.dtu.dk/services/TMHMM).

\subsection{Total RNA Isolation and cDNA Synthesis}

Total RNA was extracted from apterous adult aphids with TRIzol (Invitrogen, Carlsbad, CA, USA) according to the manufacturer's instructions and treated with RNase-free DNase I (TaKaRa, Kyoto, Japan). The RNA samples were quantified by measuring absorbance at $260 \mathrm{~nm}$; quality was assessed via agarose gel electrophoresis. First-strand cDNA was synthesized from total RNA using a PrimeScript $^{\mathrm{TM}}$ First-Strand cDNA Synthesis Kit (Takara, Japan) with oligo(dT) ${ }_{18}$.

\section{6. qRT-PCR and Data Analysis}

qRT-PCR was performed using an ABI 7500 instrument (Applied Biosystems) with SYBR ${ }^{\circledR}$ Premix Ex Taq ${ }^{\mathrm{TM}}$ II (Tli RNaseH Plus; Takara, Japan). The gene primers for qRT-PCR (Table S1) were synthesized by Sangon Biotech Co., Ltd. (Shanghai, China). The thermal cycling protocol included an initial denaturation step at $95^{\circ} \mathrm{C}$ for $30 \mathrm{~s}$ followed by 40 cycles of $95{ }^{\circ} \mathrm{C}$ for $5 \mathrm{~s}$ and $60{ }^{\circ} \mathrm{C}$ for $34 \mathrm{~s}$. The fluorescence signal was measured at the end of each extension step at $60^{\circ} \mathrm{C}$. After amplification, a dissociation program with steps of $95^{\circ} \mathrm{C}$ for $15 \mathrm{~s}, 60^{\circ} \mathrm{C}$ for $1 \mathrm{~min}$, and $95^{\circ} \mathrm{C}$ for $15 \mathrm{~s}$ was performed to confirm that only specific products were amplified. The housekeeping genes actin and para, which encodes a voltage-gated sodium channel, were used as internal reference genes for $M$. persicae nicotianae [32]. Relative gene expression was calculated with the $2^{-\Delta \Delta C T}$ method [33]. The experiment was independently performed three times for each strain. Significant differences were analyzed using GraphPad InStat 3 statistical software (GraphPad Software, 2000). 


\subsection{Artificial Diet Rearing and dsRNA Feeding}

Using DNAMAN 6.0 software, we designed specific primers based on the UGT sequences of M. persicae (Supplementary Data 1) and the possible interference sites predicted with online prediction software (http://www.dkfz.de/signaling/e-rnai3/). Gene fragments were amplified from cDNA and cloned into the pGEM-T vector (Promega, Madison, WI, USA). The amplified DNA fragments served as templates for RNA synthesis using the T7 RiboMAX ${ }^{\mathrm{TM}}$ Express RNAi System (Promega, USA). Enhanced cyan fluorescent protein $(E C F P)$ dsRNA was synthesized under identical conditions with the primers listed in Table S1. The artificial diet and the rearing method used in this study were reported previously by Peng et al. (2016) [31]. The diet (15\% sucrose) was prepared in DEPC-treated water to ensure the absence of RNase activity. For the dsRNA feeding experiments, dsRNA was added to the artificial diet at a concentration of $100 \mathrm{ng} / \mu \mathrm{L}$; an artificial diet containing dsRNA-ECFP was used as a control. Eighty apterous adult M. persicae nicotianae were transferred onto the artificial diet for rearing. For analysis of the efficiency of dsRNA knockdown of UGT expression, the aphids were fed an artificial diet containing dsRNA (100 ng/ $\mu \mathrm{L})$ for $48 \mathrm{~h}$ and then collected for qRT-PCR. To assess the sensitivity of the aphids to nicotine after RNAi of $U G T, 80$ apterous adult $M$. persicae nicotianae individuals were transferred to an artificial diet $(1 \mathrm{~mL})$ containing nicotine $(100 \mathrm{mg} / \mathrm{L})$ mixed with dsRNA-UGT $(100 \mathrm{ng} / \mu \mathrm{L})$; dsRNA-ECFP was used as the control. The mortality of the aphids was recorded after $48 \mathrm{~h}$. Each treatment included three replicates ( 80 aphids in each replicate).

\section{Conclusions}

The present study confirmed that the enzymes encoded by the identified highly-expressed UGTs may contribute to detoxification of nicotine or its primary metabolites by glycosylation and may further contribute to host plant adaptation in M. persicae nicotianae. These results contribute to our understanding of host adaptation mechanisms in M. persicae.

Supplementary Materials: The following are available online at http://www.mdpi.com/1422-0067/20/15/3637/s1. Table S1: Primers used in the experiments. Act: actin; Aph: para (a voltage-gated sodium channel); dsRNA, double-stranded RNA; F, forward; ORF, open reading frame; R, reverse. Lowercase letters indicate the T7 RNA polymerase promoter. Supplementary Data 1: M. persicae UGT transcripts. Supplementary Data 2: H. armigera and B. mori UGT sequences extracted from the UGT Nomenclature Committee database.

Author Contributions: Y.P.; P.X.; and Q.S. designed the research, analyzed the data, and wrote the paper. Y.P.; X.Z.; and X.L. carried out most of the experiments. All authors read and approved the final version of the manuscript.

Funding: This work was sponsored by the National Natural Science Foundation of China (31572024) and tobacco integrated pest management of China tobacco (110201603011).

Acknowledgments: The authors thank Michael H. Court of Washington State University for his kind help on behalf of the UGT nomenclature committee in renaming the UGTs identified in this study.

Conflicts of Interest: The authors declare that they have no conflict of interest with regard to the contents of this article.

$\begin{array}{ll}\text { Abbreviations } \\ \text { UGT } & \text { Uridine diphosphate (UDP)-glycosyltransferase } \\ \text { P450 } & \text { Cytochrome P450 monooxygenase } \\ \text { CarE } & \text { Carboxylesterase } \\ \text { GST } & \text { Glutathione S-transferase } \\ \text { qPCR } & \text { Quantitative real-time polymerase chain reaction } \\ \text { RNAi } & \text { RNA interference } \\ \text { Sul } & \text { Sulfinpyrazone } \\ \text { 5-Nul } & \text { 5-nitrouracil } \\ \text { NCBI } & \text { National Center for Biotechnology Information }\end{array}$




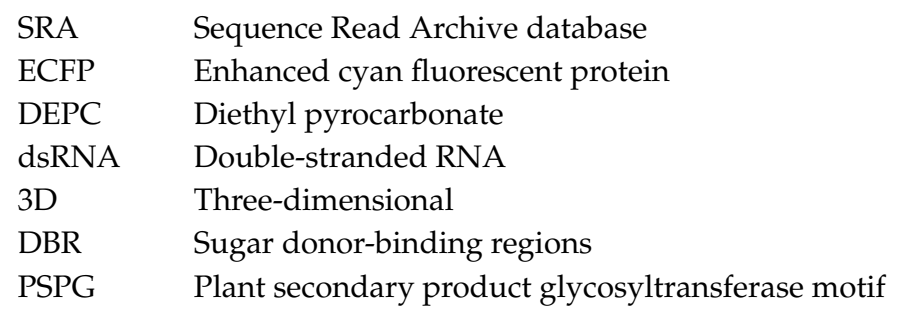

\section{References}

1. Blackman, R.L.; Eastop, V.F. Aphids on the World's Crops: An Identification and Information Guide; John Wiley \& Sons Ltd.: Chichester, UK, 2000.

2. Blackman, R.L. Morphological discrimination of a tobacco-feeding form from Myzus persicae (Sulzer) (Hemiptera: Aphididae), and a key to New world Myzus (Nectarosiphon) species. Bull. Entomol. Res. 1987, 77, 713-730. [CrossRef]

3. Margaritopoulos, J.T.; Malarky, G.; Tsitsipis, J.A.; Blackman, R.L. Microsatellite DNA and behavioural studies provide evidence of host-mediated speciation in Myzus persicae (Hemiptera: Aphididae). Biol. J. Linn. Soc. Lond. 2007, 91, 687-702. [CrossRef]

4. Cardoza, Y.J.; Wang, S.F.; Reidy-Crofts, J.; Edwards, O.R. Phloem alkaloid tolerance allows feeding on resistant Lupinus angustifolius by the aphid Myzus persicae. J. Chem. Ecol. 2006, 32, 1965-1976. [CrossRef] [PubMed]

5. $\quad$ Robertson, H.; Martos, R.; Sears, C.; Todres, E.; Walden, K.; Nardi, J. Diversity of odourant binding proteins revealed by an expressed sequence tag project on male Manduca sexta moth antennae. Insect Mol. Biol. 1999, 8, 501-518. [CrossRef] [PubMed]

6. Wilkinson, C.F. Xenobiotic conjugation in insects. In Xenobiotic Conjugation Chemistry; Paulson, G.D., Caldwell, J., Hutson, D.H., Menn, J.J., Eds.; American Chemical Society: Washington, DC, USA, 1986; pp. 48-61.

7. Li, X.; Schuler, M.A.; Berenbaum, M.R. Molecular mechanisms of metabolic resistance to synthetic and natural xenobiotics. Annu. Rev. Entomol. 2007, 52, 231-253. [CrossRef] [PubMed]

8. Snyder, M.J.; Glendinning, J.I. Causal connection between detoxification enzyme activity and consumption of a toxic plant compound. J. Comp. Physiol. A 1996, 179, 255-261. [CrossRef] [PubMed]

9. Feyereisen, R. Insect P450 enzymes. Annu. Rev. Entomol. 1999, 44, 507-533. [CrossRef]

10. Scott, J.G. Cytochromes P450 and insecticide resistance. Insect Biochem. Mol. Biol. 1999, 29 , 757-777. [CrossRef]

11. Mao, Y.; Cai, W.; Wang, J.; Hong, G.; Tao, X.; Wang, L.; Huang, Y.; Chen, X. Silencing a cotton bollworm P450 monooxygenase gene by plant-mediated RNAi impairs larval tolerance of gossypol. Nat. Biotechnol. 2007, 25, 1307-1313. [CrossRef]

12. Kliot, A.; Kontsedalov, S.; Ramsey, J.S.; Jander, G.; Ghanim, M. Adaptation to nicotine in the facultative tobacco-feeding hemipteran Bemisia tabaci. Pest Manag. Sci. 2014, 70, 1595-1603. [CrossRef]

13. Mackenzie, P.I.; Owens, I.S.; Burchell, B.; Bock, K.W.; Bairoch, A.; Bélanger, A.; Fournel-Gigleux, S.; Green, M.; Hum, D.W.; Iyanagi, T.; et al. The UDPglycosyltransferase gene superfamily: Recommended nomenclature update based on evolutionary divergence. Pharmacogenetics 1997, 7, 255-269. [CrossRef] [PubMed]

14. Heckel, D.G. Insect detoxification and sequestration strategies. Ann. Plant Rev. 2014, 47, 77-114.

15. Heidel-Fischer, H.M.; Vogel, H. Molecular mechanisms of insect adaptation to plant secondary compounds. Curr. Opin. Insect Sci. 2015, 8, 8-14. [CrossRef]

16. Ahmad, S.A.; Hopkins, T.L. Phenol b-glucosyltransferase and b-glucosidase activities in the tobacco hornworm larva Manduca sexta (L.): Properties and tissue localization. Arch. Insect Biochem. Physiol. 1992, 21, 207-224. [CrossRef]

17. Ahn, S.J.; Badenes-Pérez, F.R.; Heckel, D.G. A host-plant specialist, Helicoverpa assulta, is more tolerant to capsaicin from Capsicum annuum than other noctuid species. J. Insect Physiol. 2011, 57, 1212-1219. [CrossRef]

18. Ahn, S.J.; Badenes-Pérez, F.R.; Reichelt, M.; Svatoš, A.; Schneider, B.; Gershenzon, J.; Heckel, D.G. Metabolic detoxification of capsaicin by UDP glycosyltransferase in three Helicoverpa species. Arch. Insect Biochem. Physiol. 2011, 78, 104-118. [CrossRef] [PubMed] 
19. Wouters, F.C.; Reichelt, M.; Glauser, G.; Bauer, E.; Erb, M.; Gershenzon, J.; Vassao, D.G. Reglucosylation of the benzoxazinoid DIMBOA with inversion of stereochemical configuration is a detoxification strategy in lepidopteran herbivores. Angew. Chem. 2014, 53, 11320-11324. [CrossRef]

20. Krempl, C.; Sporer, T.; Reichelt, M.; Ahn, S.J.; Heidel-Fischer, H.; Vogel, H.; Heckel, D.G.; Joußen, N. Potential detoxification of gossypol by UDP-glycosyltransferases in the two Heliothine moth species Helicoverpa armigera and Heliothis virescens. Insect Biochem. Mol. Biol. 2016, 71, 49-57. [CrossRef]

21. Bass, C.; Zimmer, C.T.; Riveron, J.M.; Wilding, C.S.; Wondji, C.S.; Kaussmann, M.; Field, L.M.; Williamson, M.S.; Nauen, R. Gene amplification and microsatellite polymorphism underlie a recent insect host shift. Proc. Natl. Acad. Sci. USA 2013, 110, 19460-19465. [CrossRef]

22. Ramsey, J.S.; Elzinga, D.A.; Sarkar, P.; Xin, Y.R.; Ghanim, M.; Jander, G. Adaptation to nicotine feeding in Myzus persicae. J. Chem. Ecol. 2014, 40, 869-877. [CrossRef]

23. Meech, R.; Miners, J.O.; Lewis, B.C.; Mackenzie, P.I. The glycosidation of xenobiotics and endogenous compounds: Versatility and redundancy in the UDP glycosyltransferase superfamily. Pharmacol. Ther. 2012, 134, 200-218. [CrossRef] [PubMed]

24. Mao, Y.; Tao, X.; Xue, X.; Wang, L.; Chen, X. Cotton plants expressing CYP6AE14 double-stranded RNA show enhanced resistance to bollworms. Transgenic Res. 2011, 20, 665-673. [CrossRef] [PubMed]

25. Meech, R.; Mackenzie, P.I. UDP-glucuronosyltransferase, the role of the amino terminus in dimerization. J. Biol. Chem. 1997, 272, 26913-26917. [CrossRef] [PubMed]

26. Li, X.; Zhu, B.; Gao, X.; Liang, P. Over-expression of UDP-glycosyltransferase gene UGT2B17 is involved in chlorantraniliprole resistance in Plutella xylostella (L.). Pest Manag Sci. 2017, 73, 1402-1409. [CrossRef] [PubMed]

27. Li, X.; Shi, H.; Gao, X.; Liang, P. Characterization of UDP-glucuronosyltransferase genes and their possible roles in multi-insecticide resistance in Plutella xylostella (L.). Pest Manag. Sci. 2018, 74, 695-704. [CrossRef] [PubMed]

28. Pan, Y.; Tian, F.; Wei, X.; Wu, Y.; Gao, X.; Xi, J.; Shang, Q. Thiamethoxam Resistance in Aphis gossypii Glover Relies on Multiple UDP-Glucuronosyltransferases. Front. Physiol. 2018, 9, 322. [CrossRef]

29. Kaplanoglu, E.; Chapman, P.; Scott, I.M.; Donly, C. Overexpression of a cytochrome P450 and a UDP-glycosyltransferase is associated with imidacloprid resistance in the Colorado potato beetle, Leptinotarsa decemlineata. Sci. Rep. 2017, 7, 1762. [CrossRef]

30. Wang, M.; Liu, X.; Shi, L.; Liu, J.; Shen, G.; Zhang, P.; Lu, W.; He, L. Functional analysis of UGT201D3 associated with abamectin resistance in Tetranychus cinnabarinus (Boisduval). Insect Sci. 2018. [CrossRef]

31. Peng, T.; Pan, Y.; Gao, X.; Xi, J.; Zhang, L.; Ma, K.; Wu, Y.; Zhang, J.; Shang, Q. Reduced abundance of the CYP6CY3-targeting let-7 and miR-100 miRNAs accounts for host adaptation of Myzus persicae nicotianae. Insect Biochem. Mol. Biol. 2016, 75, 89-97. [CrossRef]

32. Vandesompele, J.; Preter, K.D.; Pattyn, F.; Poppe, B.; Roy, N.V.; De Paepe, A.; Speleman, F. Accurate normalization of real-time quantitative RT-PCR data by geometric averaging of multiple internal control genes. Genome Biol. 2002, 3, research0034. [CrossRef]

33. Pfaffl, M.W. A new mathematical model for relative quantification in real-time RT-PCR. Nucleic Acids Res. 2001, 29, e45. [CrossRef] [PubMed]

(C) 2019 by the authors. Licensee MDPI, Basel, Switzerland. This article is an open access article distributed under the terms and conditions of the Creative Commons Attribution (CC BY) license (http://creativecommons.org/licenses/by/4.0/). 